\title{
Relative Efficacy of Swallowing Versus Non-swallowing Tasks in Dysphagia Rehabilitation: Current Evidence and Future Directions
}

\author{
Teresa C. Drulia $\cdot$ Christy L. Ludlow
}

Published online: 25 September 2013

(c) Springer Science + Business Media New York 2013

\begin{abstract}
Clinical trials published in 2012 and the first 6 months of 2013 were reviewed. These involved either traditional dysphagia therapy, indirect methods not involving dysphagia therapy, or a combination of direct and indirect methods. Of 27 studies, 7 were RCTs, 5 were controlled clinical trials, and the remainder were uncontrolled case series. Sixteen studies combined an indirect treatment with traditional dysphagia therapy; only one study examined one technique for direct swallowing therapy. Effect sizes were computed and contrasted for each trial. Traditional dysphagia therapy had small to moderate effect sizes (between 0.3 and 0.6 ), while spontaneous recovery in acute stroke had effect sizes of 1.2. Placebo effects on patient ratings of degree of improvement on swallowing were estimated as small to moderate. To improve effect sizes, adaptive research designs are needed to develop the optimal methods and dosages of therapy before future clinical trials.
\end{abstract}

Keywords Dysphagia therapy - Strengthening exercises · Electrical stimulation - Random controlled trials .

Spontaneous recovery

\section{Introduction}

The purpose of this review was to examine the current trends and relative efficacy of different approaches to the rehabilitation of swallowing disorders. Dysphagia or swallowing

\footnotetext{
T. C. Drulia · C. L. Ludlow $(\bowtie)$

James Madison University, 801 Carrier Drive,

MSC 4304, Harrisonburg, VA 22807, USA

e-mail: ludlowcx@jmu.edu

T. C. Drulia

e-mail: druliatc@jmu.edu
}

disorders are secondary to different diseases or disorders such as stroke, aging, neurodegenerative diseases such as Parkinson's disease, and following chemo-radiation for the treatment of head and neck cancer. Approaches include: (1) training a new approach for swallowing to improve airway protection; (2) treating the primary disorder in the expectation that the secondary swallowing disorder will improve; or (3) providing less direct approaches to dysphagia either by strengthening a muscle function or providing sensory stimulation. Here, we employed the classification approach used by Zhen et al. [1], that differentiates direct swallowing therapies from indirect swallowing therapy approaches. Direct therapies (DT) include: compensatory strategies while swallowing such as chin tuck or head turn to increase airway protection, augmentative swallowing maneuvers such as Mendelsohn [2] or supraglottic swallowing, or diet modification to reduce the risk of aspiration such as thickened liquids [3]. Indirect therapies (IDT) do not directly target swallowing retraining, but are aimed at treating the underlying disease through pharmacological means, surgery, or involving muscle strengthening, sensory stimulation, or cortical stimulation by transcranial magnetic stimulation or transcranial direct current stimulation [1].

\section{Searches}

To review the therapeutic trials on dysphagia over the 1.5-year period from January 2012 to June 2013, Scopus and Medline databases were searched for studies of dysphagia therapy using search terms of: (dysphagia or swallowing disorders) and (rehabilitation or therapy) and (treatment) and (2012 or 2013), and (human). Both Mesh and text terms were searched.

Initially, 1,546 articles were retrieved and were reviewed by the first author to select clinical trials providing treatment 


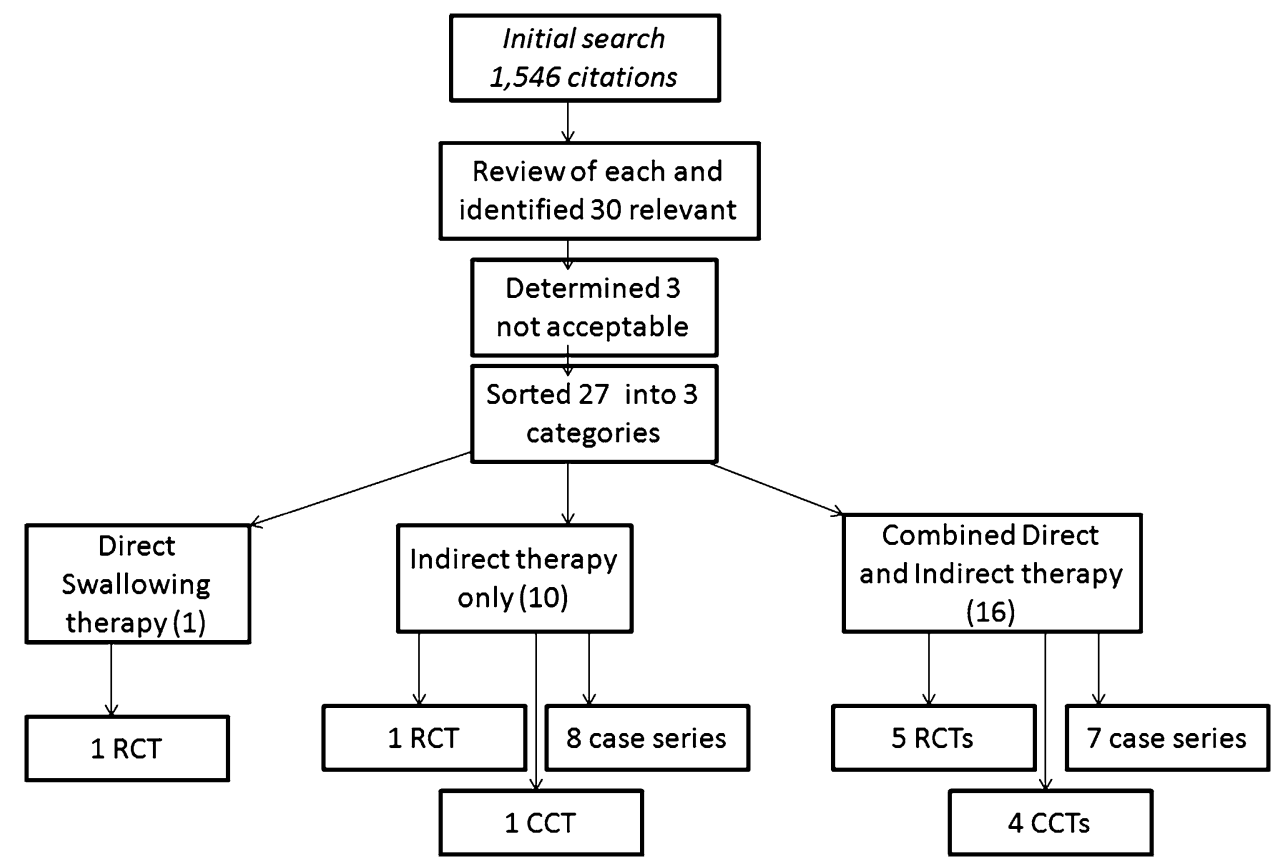

Fig. 1 The review and study classification process. Over 1,500 studies were identified for review from PUBMED and Scopus published between January 2012 and June 2013. After careful examination, 27 were selected as clinical trials involving treatment of dysphagia. Direct dysphagia therapy includes training patients on swallowing using compensatory techniques, swallowing maneuvers such as the Mendelsohn maneuver, consistency changes, and diet modification. Indirect therapy includes methods for modifying the

for patients with dysphagia. Of the initial group, 30 were selected as representing some aspect of treatment of swallowing disorders. Three studies were deleted. One only involved healthy volunteers and assessed the effects of effortful swallowing and Mendelsohn maneuver training on tongue pressure production and not swallowing [4]. Another was a study of surface electromyography measures of swallowing in a patient group with Parkinson's disease [5]. The third was a pilot study of the immediate effects of surface electrical stimulation on swallowing during videofluoroscopy in patients with Parkinson's disease and healthy volunteers [6]. After 3 deletions, 27 studies remained and are reported on here.

\section{Classification of Evidence and Measures of Efficacy}

Many clinical trials aimed at improving swallowing combined both direct and indirect approaches. Out of a total of 27 studies, we classified 1 study as involving direct swallowing retraining alone (DT), 10 studies involved nonswallowing indirect methods of treatment (IDT), and 16 examined a therapeutic regimen that combined IDT and DT methods (Fig. 1). underlying disorder causing the swallowing deficits such as surgical and neuropharmacological treatment, and sensory or cortical stimulation of persons without coincident swallowing therapy. Combined direct and indirect therapy is when an indirect technique is applied during swallowing therapy such as neuromuscular stimulation while swallowing. RCT random controlled trials, $C C T$ controlled clinical trials, case series are when no control group is employed

Random controlled trials (RCT) provided the highest level of evidence. These may involve multiple sites, include random assignment of patients to experimental and control groups, and conduct objective, blinded assessment of outcome measures. When the data on mean and standard deviations of both groups were available, we used G Power 3.1.7 [7] to estimate the effect size. Controlled studies that included a control group but did not qualify as RCTs either due to lack of random assignment to groups, unblinded assessment, or did not objectively assess outcomes were classified as controlled clinical trials (CCTs). The other studies that did not include a control group were classified as uncontrolled case series. Without an active sham or an untreated group for comparison with the treated group, the degree of benefit cannot be determined. In such studies, placebo effects or spontaneous recovery may have inflated reported treatment effects.

To measure treatment benefits Cohen's effect size [8] represents the amount of change due to the treatment relative to the amount of variance in patient scores between the two measurements. This measure can compare effects across clinical trials and does not depend on power differences as the number of patients can alter the statistical results [9]. When Cohen's $d$ effect sizes were not provided 


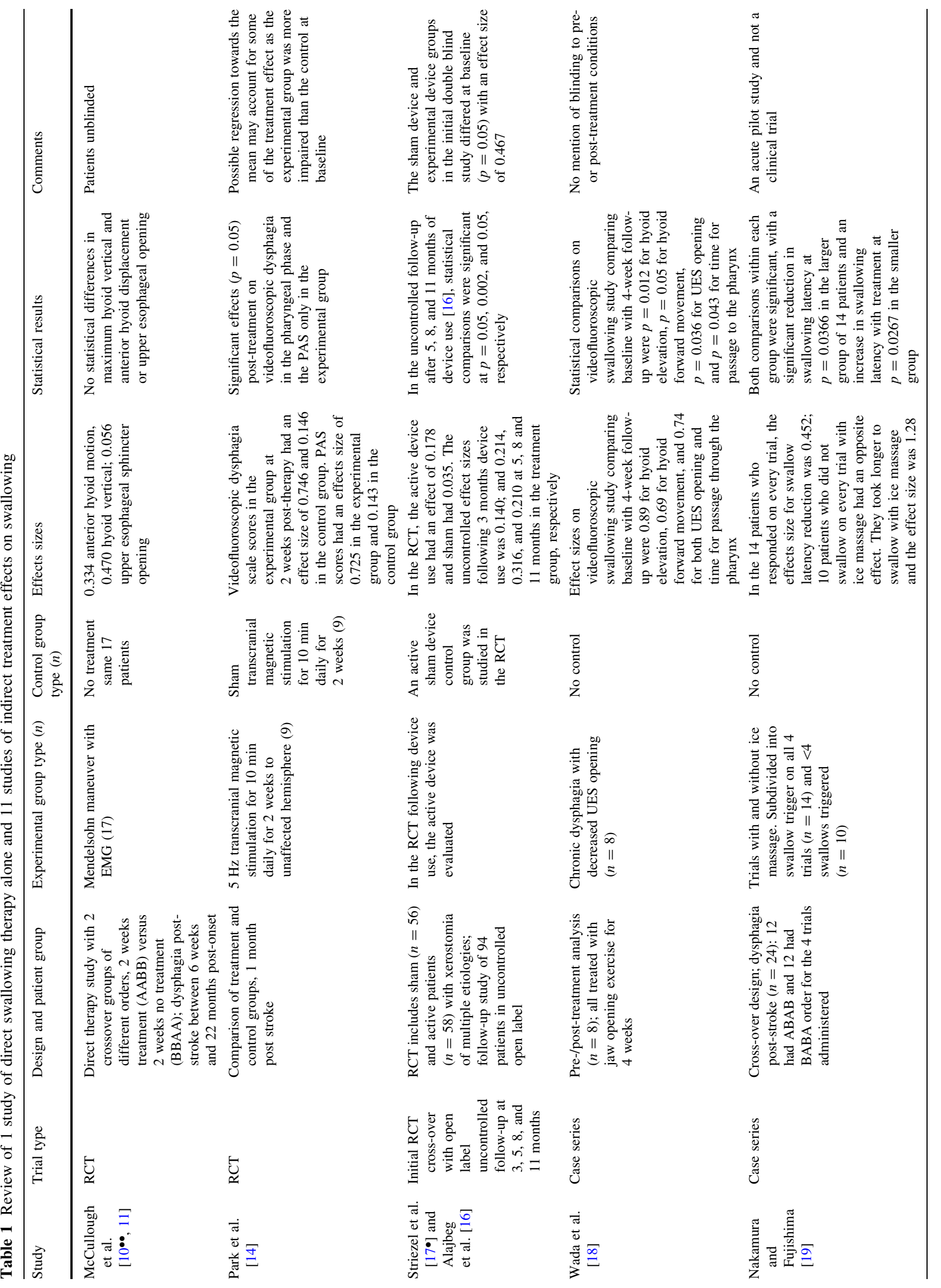




\begin{tabular}{|c|c|c|c|c|c|}
\hline & 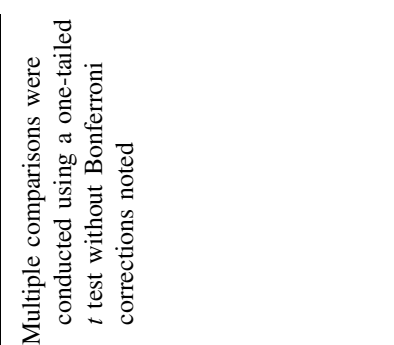 & 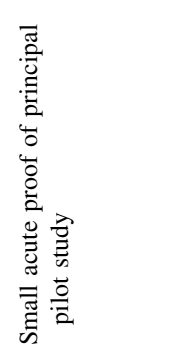 & 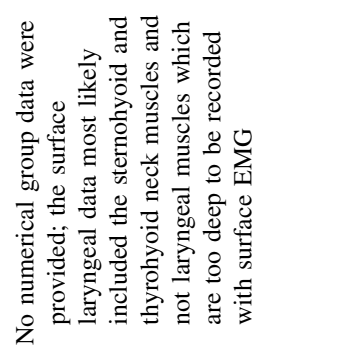 & & \\
\hline & 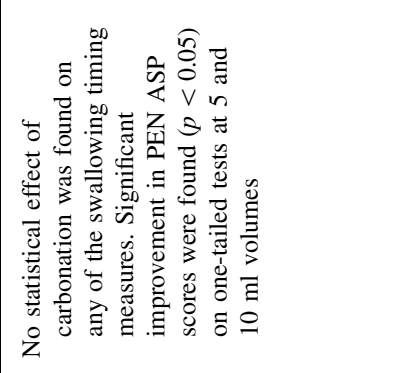 & 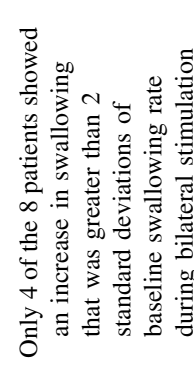 & 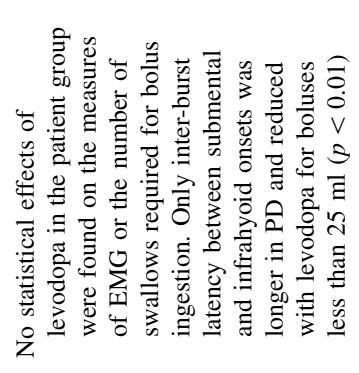 & 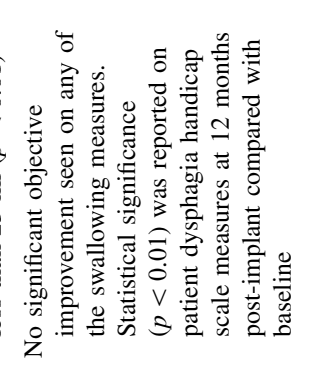 & 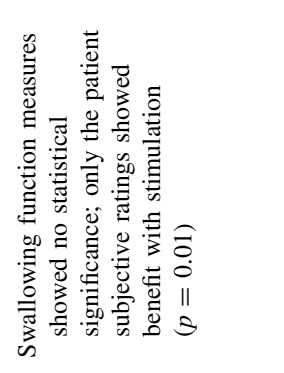 \\
\hline 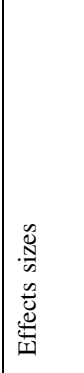 & 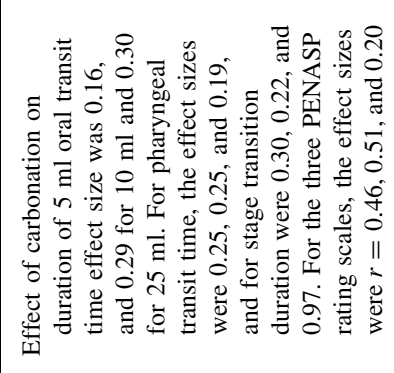 & 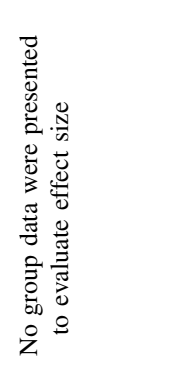 & 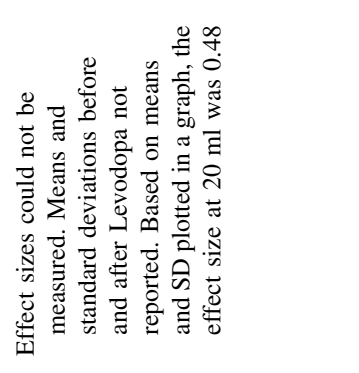 & 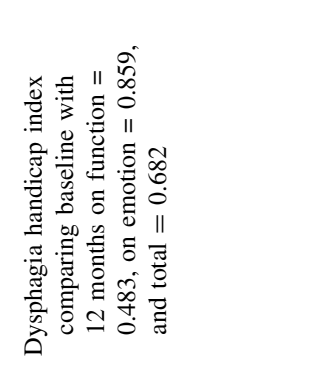 & 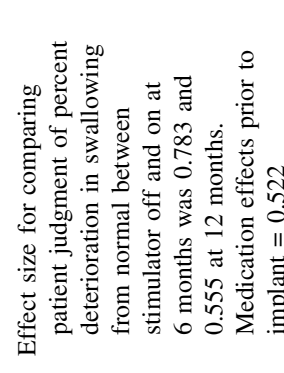 \\
\hline & $\begin{array}{l}\bar{\vdots} \\
\dot{\bar{g}} \\
\dot{0} \\
\dot{z}\end{array}$ & 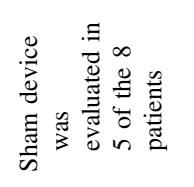 & 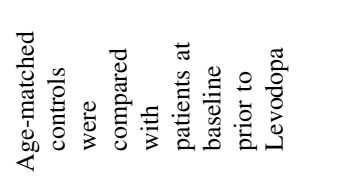 & $\begin{array}{l}\overline{0} \\
\dot{0} \\
\dot{0} \\
\dot{0}\end{array}$ & 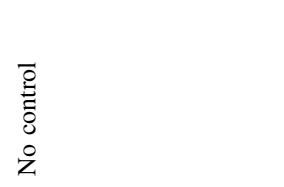 \\
\hline & 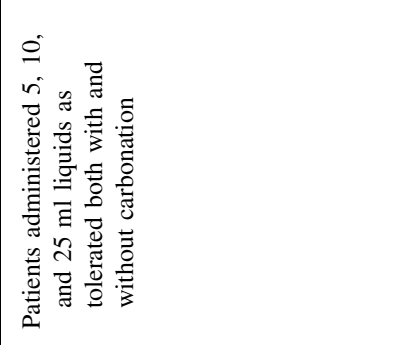 & 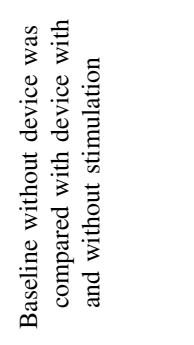 & 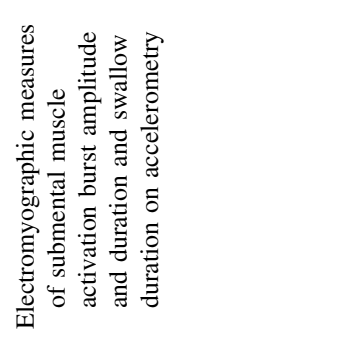 & 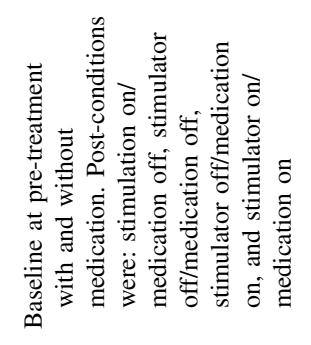 & 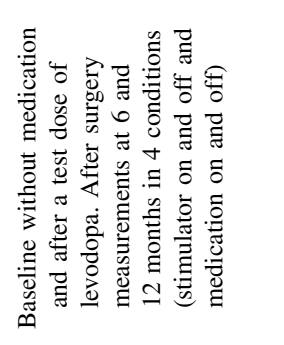 \\
\hline & 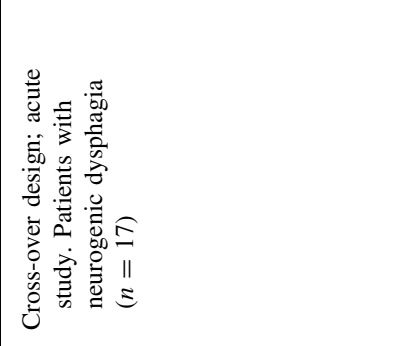 & 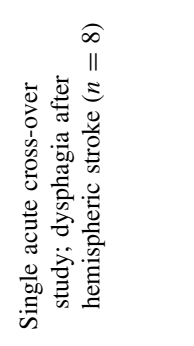 & 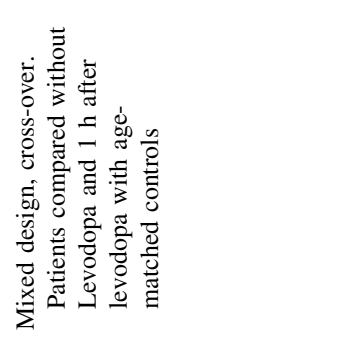 & 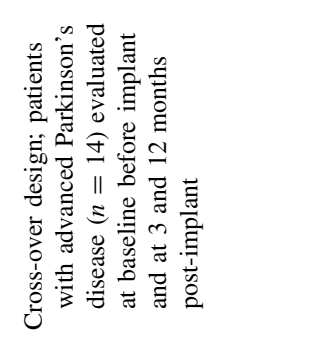 & 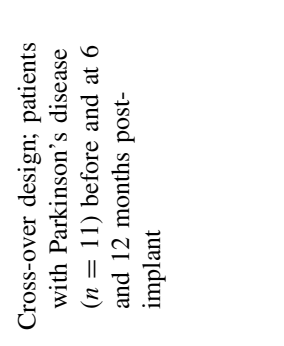 \\
\hline 总 & $\begin{array}{l}\mathscr{g} \\
\tilde{g} \\
\tilde{g} \\
\tilde{g} \\
\tilde{g}\end{array}$ & 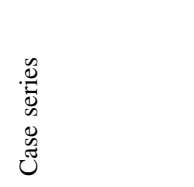 & 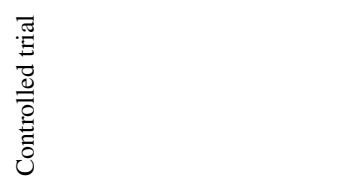 & $\begin{array}{l}\mathscr{0} \\
\tilde{\tilde{D}} \\
0 \\
\tilde{z} \\
\tilde{z}\end{array}$ & $\begin{array}{l}0 \\
\mathscr{0} \\
0 \\
0 \\
0 \\
0 \\
0\end{array}$ \\
\hline$\frac{z}{2}$ & 总 & 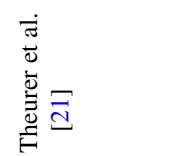 & 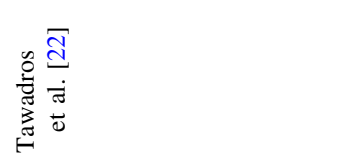 & 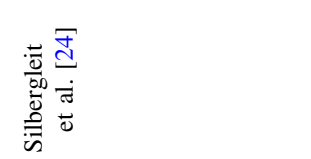 & 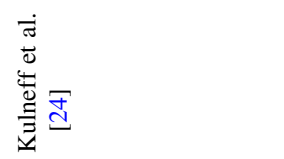 \\
\hline
\end{tabular}




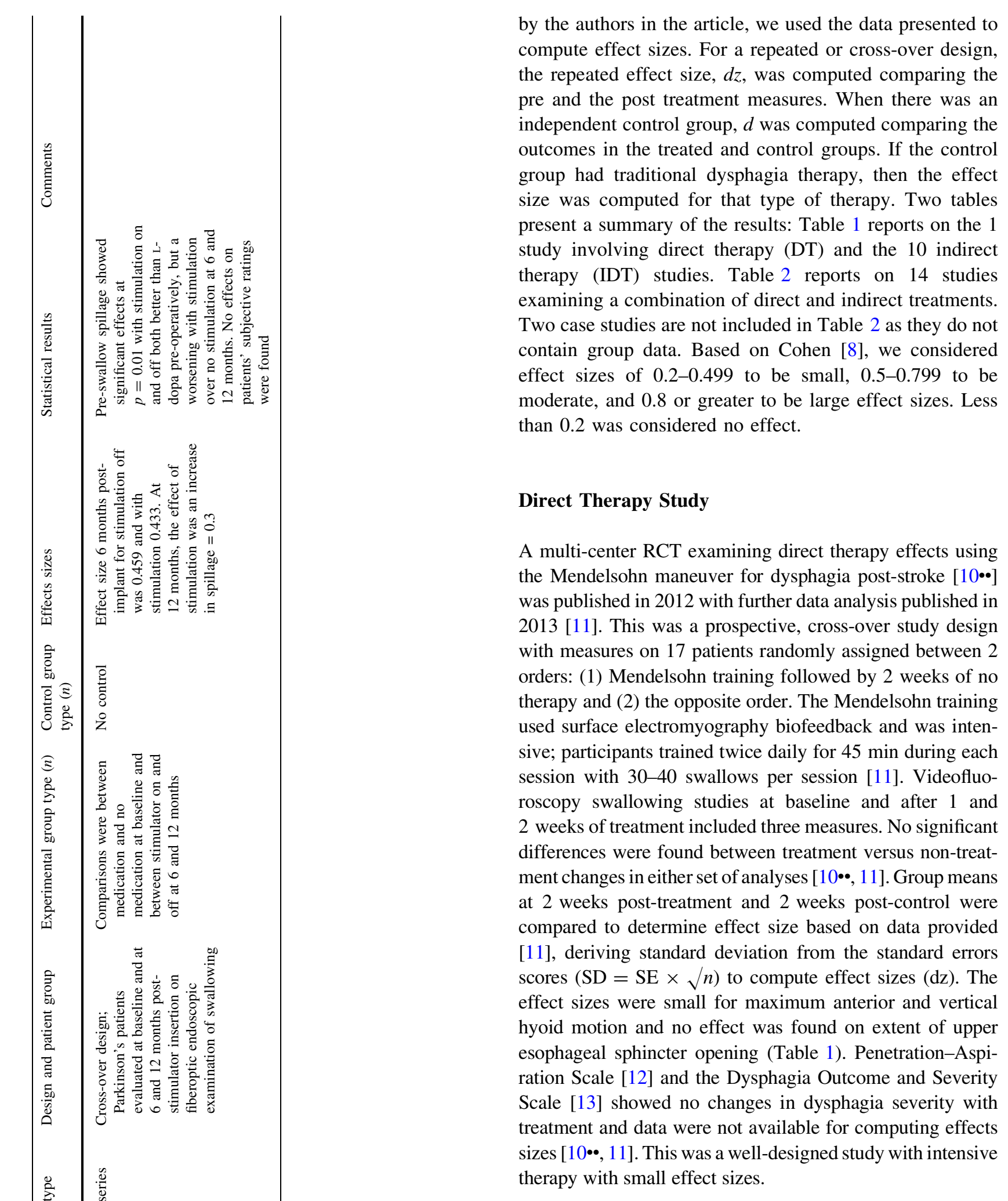

\section{Indirect Therapy Studies}

These studies employed treatment that either examined the use of a device, surgical, or pharmacological treatment of 
the disease underlying dysphagia, or used sensory or cortical stimulation in the absence of direct swallowing therapy. Two were controlled studies, eight were uncontrolled.

An RCT examined the effects of excitatory $5-\mathrm{Hz}$ transcranial magnetic stimulation over the unaffected hemisphere that might assist with recovery of swallowing [14]. The effect sizes were moderate in the experimental group (>0.7) and demonstrated no effect in the control group $(<0.2)$ on both the videofluoroscopic dysphagia scale [15] and the Penetration-Aspiration Scale [12]. However, the experimental group had a more severe videofluoroscopic dysphagia score at baseline of 33.6 while the baseline score of the control group was 23.4 which was similar to the post-treatment mean for the experimental group of 25.3. Although non-significant, the group differences at baseline represented a moderate effect size of 0.76 , similar to the effect size for treatment effects within the experimental group. The group difference at baseline may have contributed to a regression towards the mean affecting the treatment results in the experimental group but not in the control group.

The other controlled trial, an application of an intraoral electrostimulation device for treatment of xerostomia, was a large multicenter open-label, clinical trial follow-up published in 2012 [16] from a RCT conducted in 2011 [17•]. The $2011 \mathrm{RCT}$ was a multi-center prospective randomized controlled trial involving 2 stages, an initial 1-month double blind cross-over study comparing the experimental device with a sham device followed by a 3-month open-label study for the treatment of xerostomia. The electrical stimulation delivered current to the lingual nerve to enhance the salivary reflex. Swallowing difficulties were subjectively rated on a visual analogue scale $(0-100)$ by patients on their difficulty swallowing due to dry mouth. The sham device and experimental device groups in the initial double-blind study differed at baseline $(p=0.05)$ with a small effect size; the stage 1 comparison between baseline and post-treatment in the experimental group showed no effect. Similarly, no effect was found following 3 months of device use in the treatment group alone [17•]. In the uncontrolled follow-up after 5, 8, and 11 months of device use [16], although statistical comparisons within the case series of 94 patients were significant at $p=0.05,0.002$, and 0.05 , respectively, the effect sizes were all small (Table 1).

All of the remaining indirect therapy studies were uncontrolled case series. As no control group was included, placebo and spontaneous recovery effects may have contributed to the results. One study examined effects of jaw opening exercise on upper esophageal sphincter opening [18]. Effect sizes for 4 objective measures from the videofluoroscopy after 4 weeks of jaw opening exercise were moderate to large. Three studies evaluated the use of sensory stimulation before swallowing or during swallowing. In an acute pilot study, 24 patients with dysphagia post-stroke were treated using ice massage to trigger swallowing, and the latency for triggering swallowing on trials with and without ice massage was measured [19]. In the 14 patients with a swallow on every trial, the effect size of the reduction in swallowing latency was moderate. However, for the 10 patients who did not swallow on every trial, the ice massage had an opposite effect: they took longer to swallow with ice massage than without and the effect size was large. In an acute study, the effects of carbonated liquids on oropharyngeal swallowing in patients with neurogenic dysphagia and pharyngeal delay were examined using a cross-over design [20] during a videofluoroscopic swallowing study. No significant effects were found on any swallowing timing measures. All but one of the effect sizes were small indicating limited benefits. Significant changes were found on the Penetration-Aspiration Scale ratings with a moderate effect at $10-\mathrm{ml}$ volumes. A third study examined the acute effects of air pulse stimulation intra-orally on swallowing rates in 8 subjects with dysphagia post-stroke [21]. Only 4 of the 8 patients showed an increase in swallowing rates greater than 2 standard deviations of the baseline rates and no group data were provided for computing effect sizes on the 8 patients.

Four uncontrolled studies of indirect treatment in patients with Parkinson's disease have been published; one examined the effects of Levodopa on swallowing [22] in patients with Parkinson's disease and three studies examined the effects of deep brain stimulation on swallowing in Parkinson's disease [23-25]. The Levodopa study showed limited effects on submental and infrahyoid electromyographic measures; only the intra-burst onset latencies were reduced with Levodopa. Effect sizes based on the means and standard deviations plotted, were small.

Deep brain stimulation implant into the subthalamic nucleus was evaluated in 12 patients at 3 and 12 months post-implant in 4 conditions combining medication on and off and stimulator on and off [25]. Results revealed no significant changes in swallowing function on objective measures taken from the videofluorographic swallowing study; but some improvement was reported by patients on the Dysphagia Handicap Index [26] with small effects on swallowing function, high effects on emotion; and moderate effects on overall score compared with baseline at 3 and 12 months post-implant. The authors raised the point that some of these changes in patient perceptions in the absence of objective changes in swallowing may represent placebo effects.

Another study examined deep brain stimulation in Parkinson's disease in the subthalamic nucleus [24]. No benefits were found on measures of swallowing function from videofluoroscopy ratings, but patients reported a moderate 


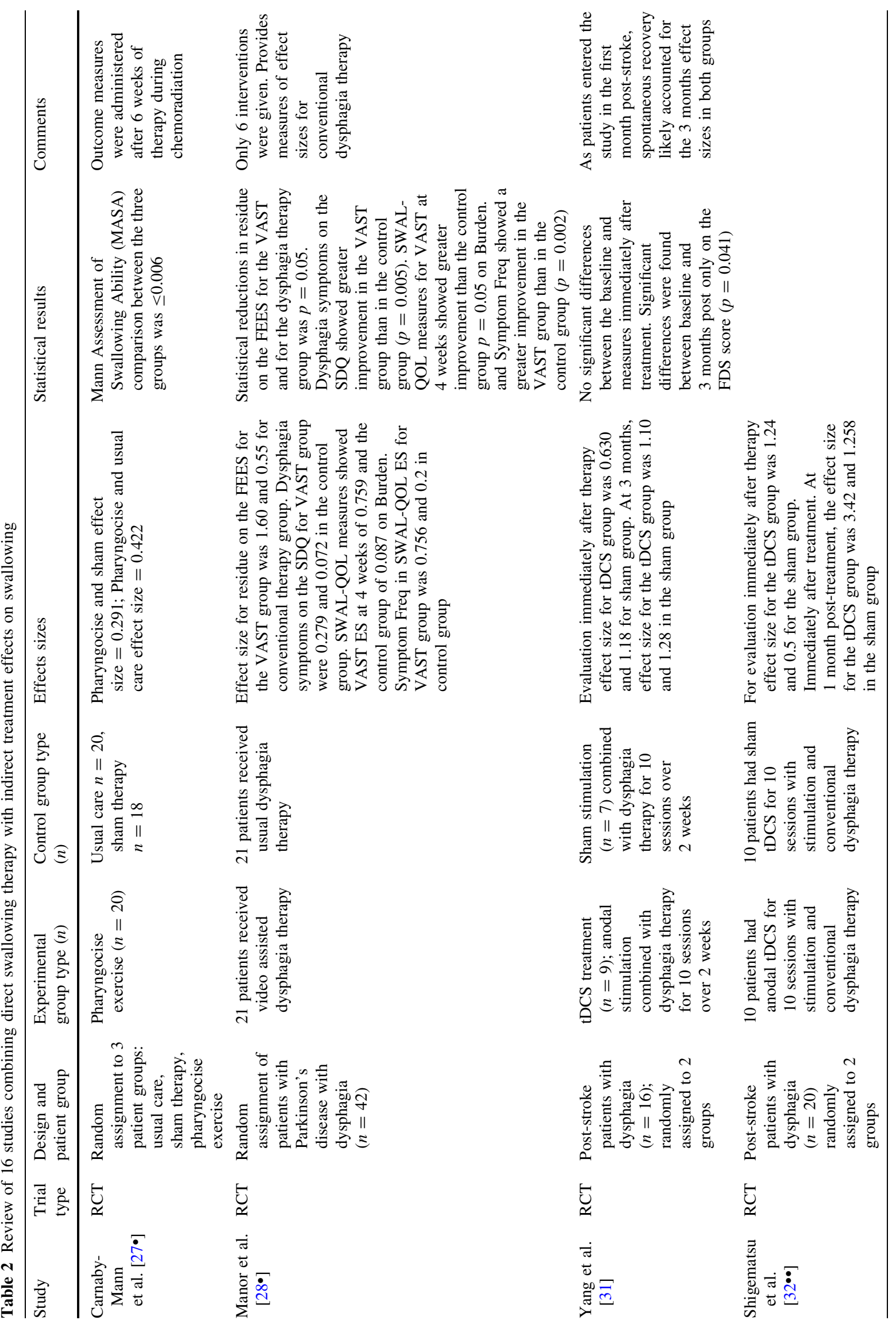




\begin{tabular}{|c|c|c|c|}
\hline & \multicolumn{3}{|c|}{ 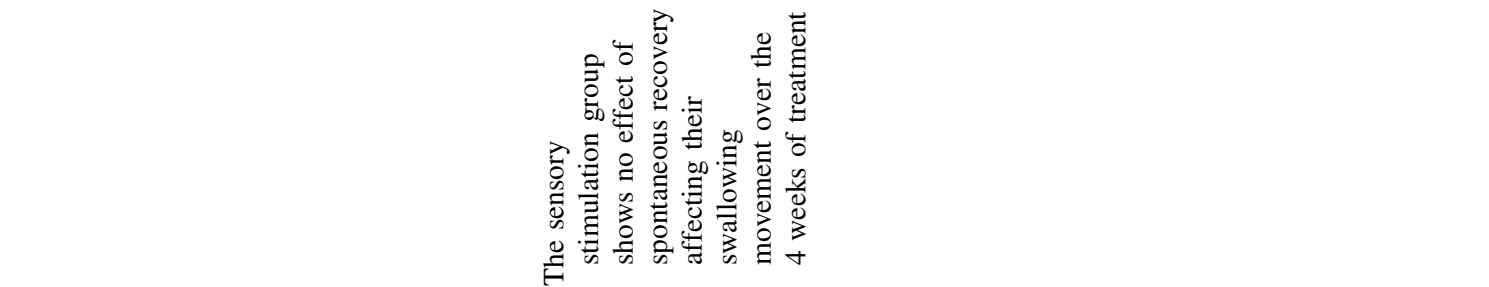 } \\
\hline & 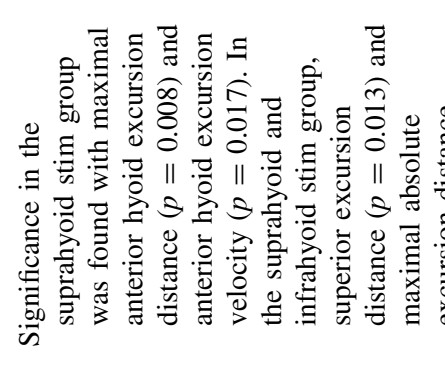 & 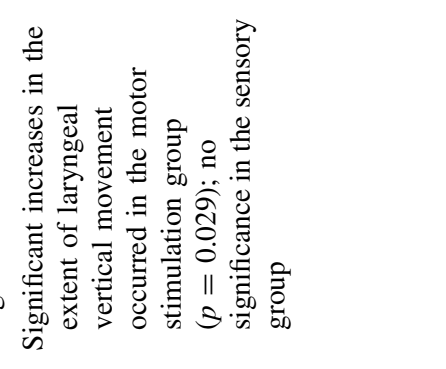 & 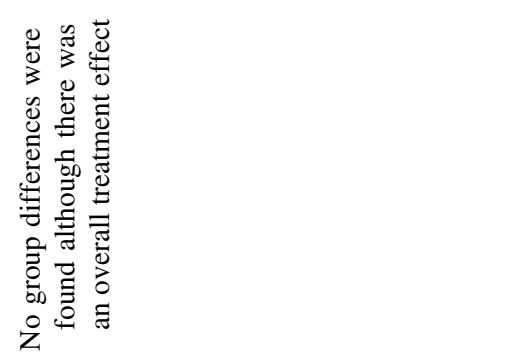 \\
\hline & 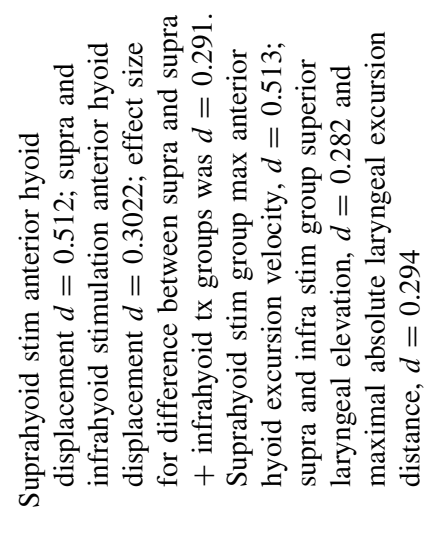 & 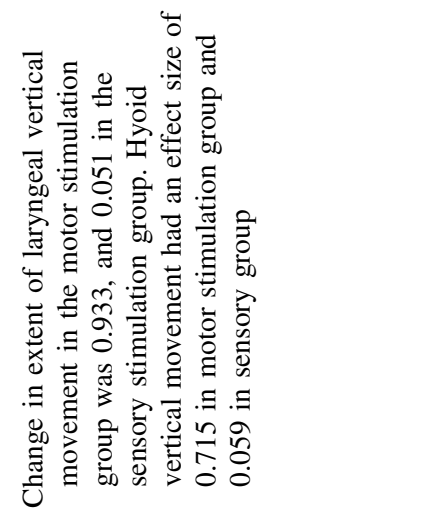 & 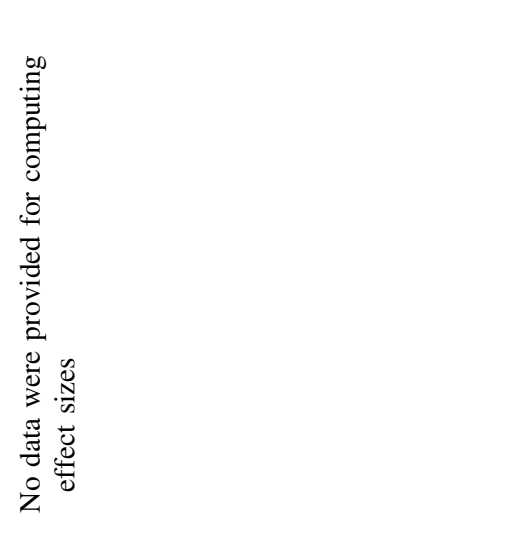 \\
\hline 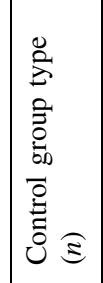 & 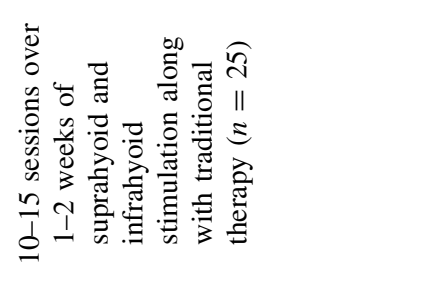 & 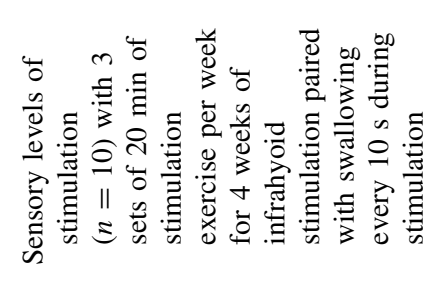 & 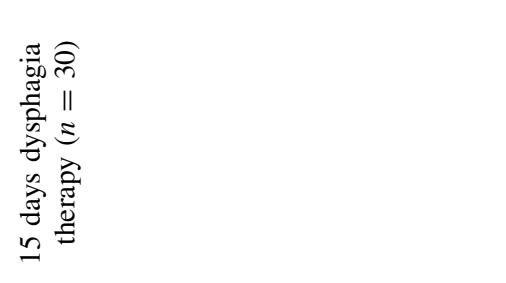 \\
\hline 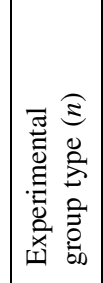 & 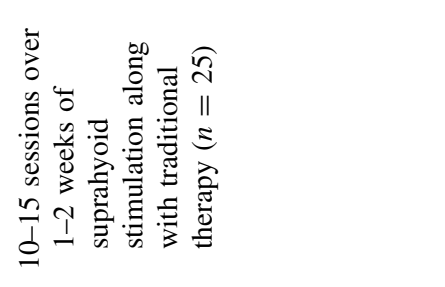 & 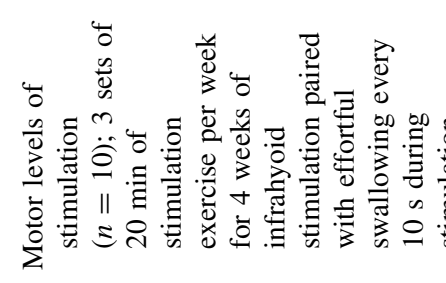 & 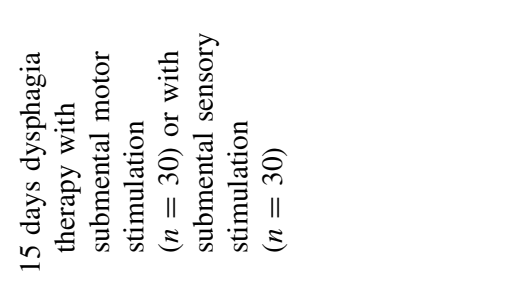 \\
\hline 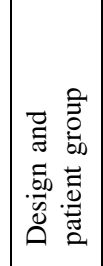 & 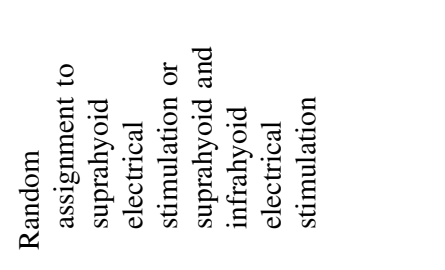 & 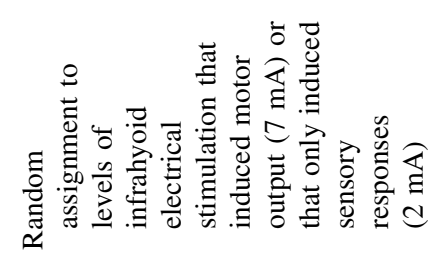 & 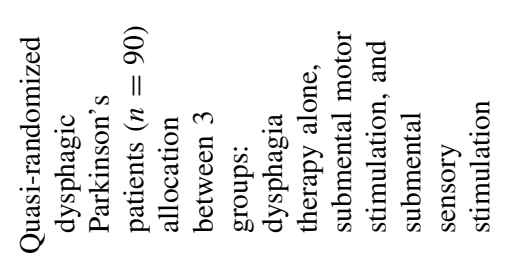 \\
\hline D્ & 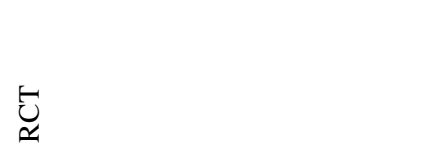 & $\approx$ & $\begin{array}{l}\text { 总 } \\
\overline{\overline{\bar{g}}} \\
\bar{g} \\
\bar{g}\end{array}$ \\
\hline & 镸离 & 总焉它 & : \\
\hline
\end{tabular}




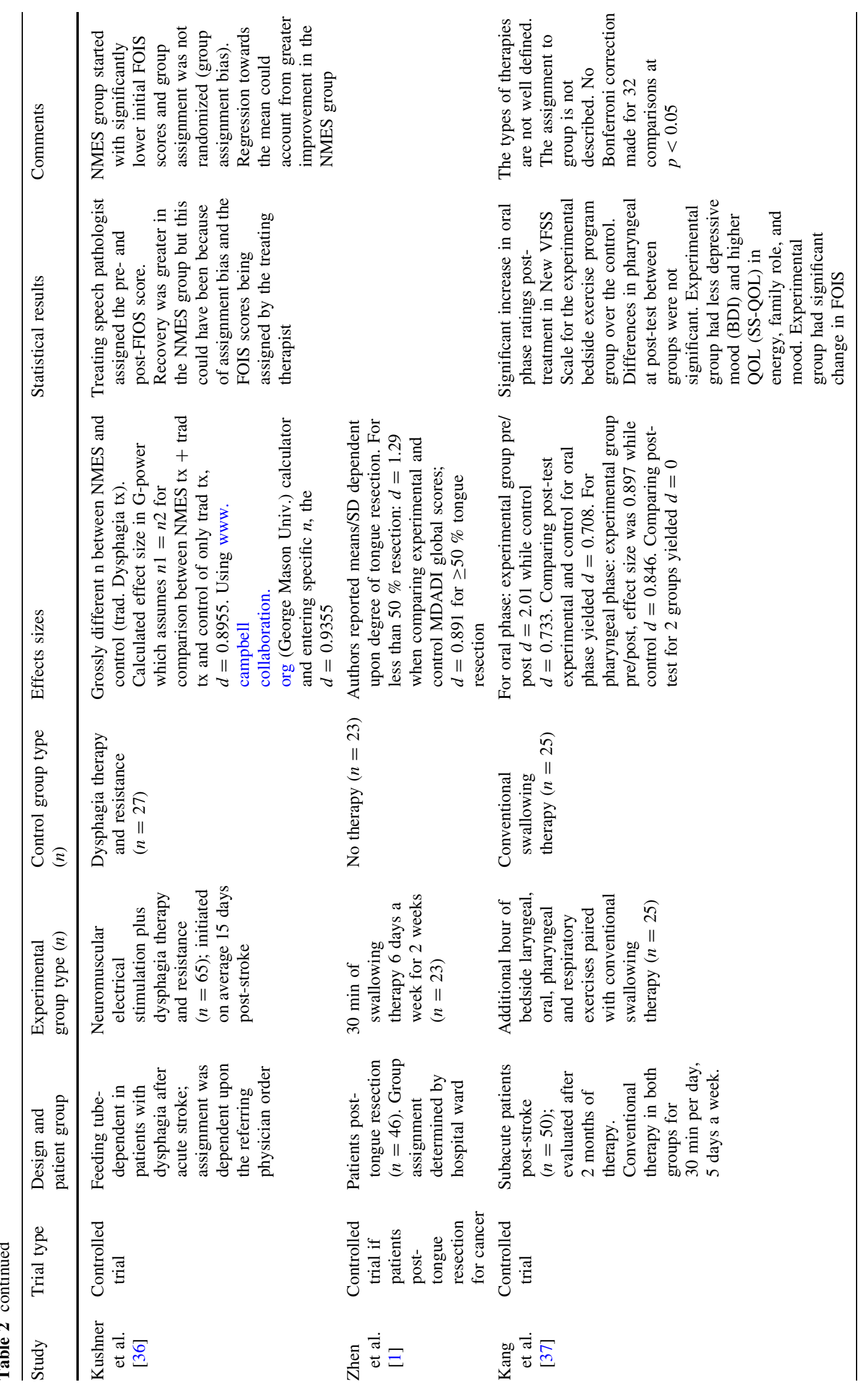




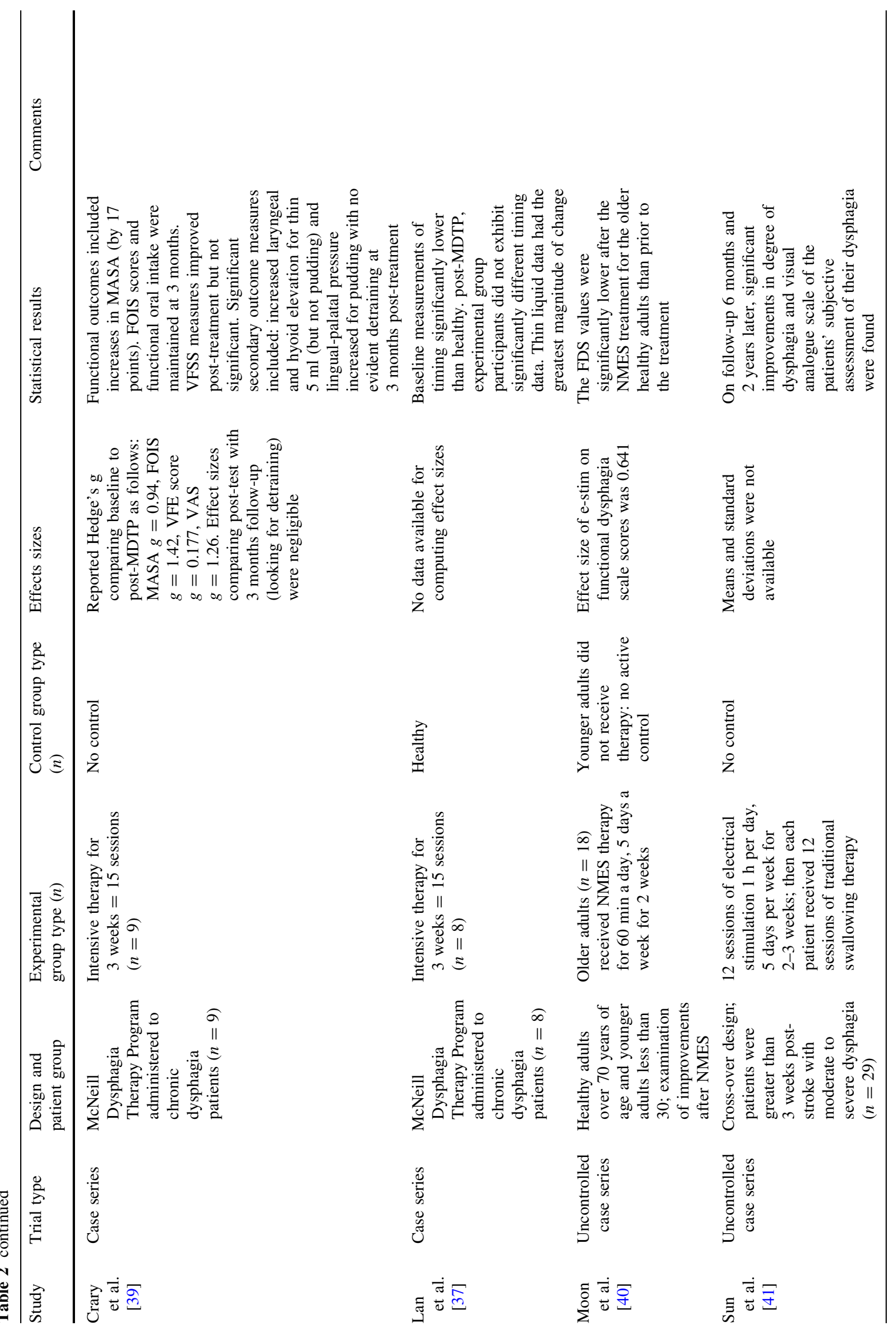


effect size of stimulation benefit on their swallowing at 6 and 12 months which may include a placebo effect. A third study of the effects of deep brain stimulation on swallowing in Parkinson's disease determined if there were deficits with stimulation in the Zona Incerta [23]. No effects were found except for a small worsening in preswallow spillage with stimulation on at 12 months postimplant; no subjective effects were found on patient ratings.

Overall, the two controlled studies of indirect treatment approaches had small or moderate treatment effects while the 8 uncontrolled studies varied with large effects only seen in patients performing jaw opening exercises; however, as this study was uncontrolled, the results cannot be distinguished from potential placebo effects. The two studies of the effects of subthalamic nucleus stimulation on swallowing in Parkinson's disease showed no benefits on objective measures of swallowing. The only benefits were in patient subjective ratings which are particularly susceptible to placebo effects after implantation and were small to moderate effects.

\section{Combined Direct and Indirect Therapy Studies}

Sixteen studies combined indirect therapy with direct swallowing therapy. Some included controls with direct therapy or a sham with direct therapy. Of these studies, five were RCTs, four were CCTs, and the remaining seven were uncontrolled case series. The combined approach was the most frequent approach in research on dysphagia treatment in recent years. This may be based on the assumption that if direct therapy shows small to moderate effect sizes then adding further therapeutic approaches will augment the results.

An RCT on a therapy described as "pharyngocise" evaluated whether active exercise combined with usual care for patients with head and neck cancer undergoing chemoradiation therapy (CRT) is beneficial [27•]. Strengthening exercise combined with swallowing therapy was compared with a sham therapy group (active controls) and a usual care group which included supervision and precautions for safe swallowing with positioning and slowed rate of feeding by the hospital speech pathology service. Both active treatment groups were seen twice daily for $45 \mathrm{~min}$ of exercise for 6 weeks. The sham treatment received a buccal extension maneuver while the pharyngocise group received jaw resistance/strengthening using the Therabite Jaw Motion Rehabilitation System. As all patients consumed a normal diet at baseline, effect size differences on the Mann Assessment of Swallowing Ability (MASA) between the three independent groups were determined at 6 weeks. The effect sizes were small between pharyngocise and sham therapy and between pharyngocise and usual care.
Another RCT compared patients with Parkinson disease and swallowing difficulties who were randomly assigned to two groups: usual dysphagia therapy $(n=21)$ and videoassisted dysphagia therapy $(n=21)$ showing videos of the typical swallowing process and patients' own swallowing as part of regular therapy during all six interventions [28•]. A large effect size for residue reduction on FEES examination in the video-assisted group compared with a moderate effect size in the usual dysphagia therapy group. Patient responses on the SDQ questionniare [29] only showed a small effect improvement and no effect in the controls. Difference in effect size occurred on scores in the video and conventional therapy group at 4 weeks and 8 months; at least moderate effect sizes occurred in the video group compared with no or small effects in the conventional therapy group on the SWAL_QOL [30]. This study is notable as the effect size of the experimental therapy was large (1.6) when compared with the moderate effect size (0.55) of conventional dysphagia therapy on residue after a swallow.

Two studies examined the combination of transcranial direct current stimulation (tDCS) combined with swallowing training post-stroke [31, 32••]. Both compared anodal tDCS with sham stimulation during ten trials of dysphagia therapy. One study used the DOSS [13, 32••] for pre- and post-measures of dysphagia while the other used the Functional Dysphagia Scale (FDS) $[15,30]$. In the first study [31], the effect size immediately after treatment in the tDCS group was moderate and high in the sham group. At 3 months post-therapy, both groups had a high effect size. Mean days post-stroke was 25.9 days $(\mathrm{SD}=10.2$ ) so most patients were within the first month post-stroke on entering the study and spontaneous recovery could account for partial improvement in both groups, particularly 3 months after therapy. In the second study [32••], patients were at least 1 month post-stroke. After 10 sessions of therapy, the tDCS group had a large effect size while the sham group had a moderate effect size, similar to the effect of usual dysphagia therapy reported for traditional therapy. Thus, after 10 sessions of traditional dysphagia therapy along with tDCS in patients seen within 1 month poststroke, both studies had an immediate effect size of 0.6 or greater. However, the post-therapy follow-up on 3 months in one study and 1 month in the second both showed a high effect size in the sham group suggesting that spontaneous recovery can contribute an effect size of 1.2 or greater.

Two other RCTs evaluated a combination of transcutaneous electrical stimulation [33, 34]. In the first study, 50 subacute stroke or brain injury patients were randomly assigned; 25 to suprahyoid stimulation only and the other 25 to suprahyoid and infrahyoid stimulation for 10-15 sessions over 2-3 weeks. Electrical stimulation was combined with traditional dysphagia therapy including chin 
tuck, multiple swallowing, effortful swallowing, supraglottic swallowing, Shaker's exercise and the Mendelsohn maneuver. All of the effect sizes evaluating objective measures of movement excursion of the hyoid and larynx in each of the two groups between before and after therapy were less than 0.52 . No differences in the treatment effects were found between the suprahyoid and infrahyoid groups. When objective movement changes were measured after therapy in subacute patients, the effect sizes were more modest (0.5) than when patients were studied in the first month post-stroke or -brain injury. The second study [34] using electrical stimulation tested the effect of effortful swallow therapy combined with either motor levels of infrahyoid stimulation or sensory levels of infrahyoid stimulation. Twenty patients were randomly assigned, 10 to each treatment group. Here, the patients were required to be more than 1 month post-stroke and three sets of exercise for 20 min each week were provided for 4 weeks. Using data provided in the paper, a high effect size occurred with motor stimulation on the extent laryngeal vertical movement and a moderate effect size for hyoid extent of vertical movement in the motor group. No effects occurred in the sensory group indicating that spontaneous recovery was not contributing significantly to the results.

Four studies were controlled without random assignment between the experimental and control groups, not qualifying as RCTs. A study combining surface electrical stimulation in three groups of Parkinson's disease with dysphagia used quasi-random allocation to three groups [35]. All received daily treatment for 15 days of dysphagia therapy with two groups also receiving surface submental electrical stimulation; one at a motor level and the other at a sensory level. No data were provided to compute effect sizes as odds ratio were provided. The statistical analysis showed no differences between treatment groups, although there was an overall treatment benefit indicating that differences in electrical stimulation did not alter the treatment outcome. Another study added electrical stimulation to traditional dysphagia therapy along with progressive resistance therapy $(n=65)$ for comparison with dysphagia therapy along with progressive resistance therapy without electrical stimulation $(n=27)$ [36]. Physician referral assigned patients to either treatment group; the NMES group was more impaired at baseline suggesting assignment bias, and regression towards the mean may have affected recovery in that group. Further FIOS scores were assigned by the treating speech therapist pre and posttreatment, also a likely source of bias.

A controlled trial compared inter-disciplinary swallowing therapy exercises in 23 patients post-tongue resection with a control group $(n=23)$ receiving no training [1]. Assignment was based on patient ward assignment with two floors of eight wards each assigned to the experimental and control groups. Therapy consisted of $30 \mathrm{~min}$ of swallowing therapy 6 days a week for 2 weeks. High effect sizes were reported when outcomes were compared between the treatment and control groups with similar degrees of tongue resection.

The addition of a bedside exercise program to conventional swallowing therapy in subacute patients $<6$ months post-stroke was evaluated in a controlled trial [37]. The addition of bedside laryngeal, oral, pharyngeal, and respiratory exercises to conventional therapy was evaluated after 2 months of conventional swallowing therapy in both groups. A high effect size was found on oral phase improvements in the exercise group while the conventional therapy group had a moderate effect size. In the pharyngeal phase, both groups had a high effect size and there were no group differences in benefit.

Six uncontrolled studies were published in the 2012-2013 period examining treatment effects of a combined regimen. Some examined proprietary treatment programs and are difficult to evaluate as the intensive therapy program is not clearly described. It may include home therapy and professional therapy with exercises, direct dysphagia therapy and NMES $[38,39]$. The patients had chronic dysphagia, however, placebo effects cannot be determined and both studies include multiple comparisons with no Bonferoni corrections. Subjective measures show large effect sizes while objective measures from videofluoroscopy show no significant improvement [38]. A case series evaluated the use of electrical stimulation in normal adults over 70 years of age on a functional dysphagia scale with significant improvement and moderate effect sizes [40]. Another study assessed swallowing after 12 sessions of electrical stimulation with dysphagia therapy followed by 12 sessions of traditional swallowing therapy [41]. Finally, two case studies were reported, one reporting using electrical stimulation for dysphagia in Wilson's disease [42] and another using manual facilitation with deep massage of the submental and infrahyoid regions to help initiate swallowing [43], along with oral sensory stimulation and Mendelsohn maneuver in 3 severely impaired dysphagia patients unresponsive to verbal commands poststroke. All increased their ability to initiate a swallow. As there were no group data to be analyzed, these case studies are not reported in Table 2.

\section{Discussion}

The number of studies evaluating therapy for dysphagia in a 1.5-year period was impressive. Most studies were aimed at augmenting traditional dysphagia therapy by adding therapy components such as electrical stimulation to traditional therapy. Few, if any, RCTs have evaluated the 
effects of traditional dysphagia therapy alone in particular patient populations. Traditional dysphagia therapy usually combines compensatory approaches such as chin tuck and head turn, effortful swallowing, Mendelsohn maneuver, thickened liquids, and supraglottic swallowing [3]. We could not find previous RCTs in the literature comparing a non-treatment control with traditional dysphagia therapy to determine the effect size to expect for traditional therapy alone. In this review, some studies used a traditional therapy group as a control and reported effect sizes ranging from 0.3 to 0.7 , which are small to moderate. Except for studies carried out in the early spontaneous recovery period post-stroke, most effect sizes for dysphagia therapy alone are small to moderate. That may be the basis for many clinical studies combining many therapy approaches in order to augment effect sizes.

Many studies showed no benefits on objective measures of swallowing, while patient subjective ratings showed small to moderate effects, which may contain placebo effects. Patients often assume that if they are undergoing treatment they are improving. Therefore, a well-defined control group is needed when using patient ratings, as both groups may show gains that are small to moderate reflecting placebo effects [25].

Another notable finding in patients seen early poststroke is that therapy effects are usually moderate when measured immediately after a few weeks of therapy. However, on follow-up 1 month later in one study and 3 months in another, both showed a similar high effect size in the sham group suggesting that spontaneous recovery can contribute a high effect size of 1.2 or greater. The large effect size for spontaneous recovery relative to treatment effect sizes present a significant difficulty when designing studies of treatment effects in acute stroke.

The vast majority of the treatment studies used 1 session of 30-60 min daily for 2 weeks for a total of 10 sessions of therapy in the experimental group. However, no studies have examined the optimal session duration, number of sessions, and time period of therapy necessary for effecting change in swallowing behavior. Many of these treatment studies may not have an adequate duration or intensity to effect change. This needs to be determined before embarking on expensive and time-consuming RCTs. Continual reassessment designs can be used to determine the optimal treatment parameters, require fewer participants, and are relatively short in duration to determine the optimal treatment parameters before embarking on a large multicenter RCT.

O'Quigley et al. [44] and associates introduced the concept of the continual reassessment method when designing clinical trials for cancer treatment. In such studies, small reiterative studies are sequenced so that ongoing data analysis "learns" from the results of particular therapy regimens in a small number of patients and adjusts the next phase treatment to be more effective and less adverse. Such trials can be blinded, with only the statistician able to analyze the results in a continuous fashion to make treatment decisions. The study proceeds with small numbers of patients at a time (3-5 participants), and the treatment parameters are readjusted in a reiterative fashion based on the data learned after each patient, and the dose response curve is remodeled [45]. This method of adjusting to find the optimal treatment dosage at the highest maximal tolerated dose can provide an effective treatment regimen with the least number of adverse events. Some of these designs might be very useful for determining the optimal dosage and intensity of a therapy before embarking on a controlled trial.

\section{Conclusions}

To date, the treatment effects in dysphagia are small to moderate in contrast to the much larger effects of spontaneous recovery in acute stroke. Objective measures of swallowing kinematics generally show limited change, while patient ratings are often confounded by placebo effects. Usually, dysphagia therapy has small to moderate effect sizes; thus, the field is actively pursuing combined treatment approaches to improve outcomes. To date, some of the highest effect sizes reported in RCTs are for cortical stimulation combined with dysphagia therapy in acute stroke. However, spontaneous recovery may be contributing to a greater degree than therapy to dysphagia recovery post-stroke.

\section{Compliance with Ethics Guidelines}

Conflict of Interest T.C. Drulia declares no conflicts of interest. C.L. Ludlow works for an institution that has received NIH grants; and consulting fees, travel expenses, patent income, and fees for developing educational presentations from Passy Muir, Inc.

Human and Animal Rights and Informed Consent This article does not contain any studies with human or animal subjects performed by any of the authors.

\section{References}

Papers of particular interest, published recently, have been highlighted as:

- Of importance

- Of major importance

1. Zhen Y, Wang JG, Tao D, Wang HJ, Chen WL. Efficacy survey of swallowing function and quality of life in response to therapeutic intervention following rehabilitation treatment in dysphagic tongue cancer patients. Eur J Oncol Nurs. 2012;16(1):54-8. 
2. Mendelsohn MS, McConnel FM. Function in the pharyngoesophageal segment. Laryngoscope. 1987;97(4):483-9.

3. Logemann JA. Evaluation and treatment of swallowing disorders. 2nd ed. Austin: Pro-Ed; 1998.

4. Fukuoka T, Ono T, Hori K, et al. Effect of the effortful swallow and the Mendelsohn maneuver on tongue pressure production against the hard palate. Dysphagia. 2013. doi:10.1007/s00455013-9464-y.

5. Ws Coriolano MDG, R Belo L, Carneiro D, et al. Swallowing in patients with Parkinson's disease: a surface electromyography study. Dysphagia. 2012;27(4):550-5.

6. Baijens LWJ, Speyer R, Passos VL, Pilz W, Roodenburg N, Clavé $P$. The effect of surface electrical stimulation on swallowing in dysphagic Parkinson patients. Dysphagia. 2012;27(4):528-37.

7. Faul F, Erdfelder E, Buchner A, Lang AG. Statistical power analyses using $G^{*}$ Power 3.1: tests for correlation and regression analyses. Behav Res Methods. 2009;41(4):1149-60.

8. Cohen J. Statistical power for the behavioral sciences. 2nd ed. Hillasdale: Erlbaum; 1988.

9. Fleiss JL. The design and analysis of clinical experiments. New York: Wiley; 1999.

10. • McCullough GH, Kamarunas E, Mann GC, Schmidley JW, Robbins JA, Crary MA. Effects of Mendelsohn maneuver on measures of swallowing duration post stroke. Top Stroke Rehabil. 2012;19(3):234-243. An RCT design using a randomized crossover method comparing the Mendelsohn maneuver (a direct therapy treatment) with no treatment in 2 different orders. Physiological measurements of anterior and vertical hyoid movement during swallowing showed a small treatment effect and statistical significance was not reached.

11. McCullough GH, Kim Y. Effects of the Mendelsohn maneuver on extent of hyoid movement and UES opening post-stroke. Dysphagia. 2013. doi:10.1007/s00455-013-9461-1.

12. Rosenbek JC, Robbins JA, Roecker EB, Coyle JL, Wood JL. A penetration-aspiration scale. Dysphagia. 1996;11(2):93-8.

13. O'Neil KH, Purdy M, Falk J, Gallo L. The Dysphagia Outcome and Severity Scale. Dysphagia. 1999;14(3):139-45.

14. Park JW, Oh JC, Lee JW, Yeo JS, Ryu KH. The effect of $5 \mathrm{~Hz}$ high-frequency rTMS over contralesional pharyngeal motor cortex in post-stroke oropharyngeal dysphagia: a randomized controlled study. Neurogastroenterol Motil. 2013;25(4):324-30.

15. Han TR, Paik NJ, Park JW, Kwon BS. The prediction of persistent dysphagia beyond six months after stroke. Dysphagia. 2008;23(1):59-64.

16. Alajbeg I, Falcão DP, Tran SD, et al. Intraoral electrostimulator for xerostomia relief: a long-term, multicenter, open-label, uncontrolled, clinical trial. Oral Surg Oral Med Oral Pathol Oral Radiol. 2012;113(6):773-81.

17. - Strietzel FP, Lafaurie GI, Mendoza GR, et al. Efficacy and safety of an intraoral electrostimulation device for xerostomia relief: a multicenter, randomized trial. Arthritis Rheum. 2011;63(1):180-190. A large multi-center RCT compared an intraoral electrical stimulation to a sham in a cross-over design; xerostomia patients' perception of swallowing difficulty was evaluated. No treatment effects were evident at 1 month in the controlled study; however, an open-label follow-up reported small treatment effects at 5, 8, and 11 months post-treatment.

18. Wada $\mathrm{S}$, Tohara $\mathrm{H}$, Iida $\mathrm{T}$, Inoue $\mathrm{M}$, Sato $\mathrm{M}$, Ueda K. Jawopening exercise for insufficient opening of upper esophageal sphincter. Arch Phys Med Rehabil. 2012;93(11):1995-9.

19. Nakamura T, Fujishima I. Usefulness of ice massage in triggering the swallow reflex. J Stroke Cerebrovasc Dis. 2013;22(4):378-82.

20. Sdravou K, Walshe M, Dagdilelis L. Effects of carbonated liquids on oropharyngeal swallowing measures in people with neurogenic dysphagia. Dysphagia. 2012;27(2):240-50.
21. Theurer JA, Johnston JL, Fisher J, et al. Proof-of-principle pilot study of oropharyngeal air-pulse application in individuals with dysphagia after hemispheric stroke. Arch Phys Med Rehabil. 2013;94(6):1088-94.

22. Tawadros PB, Cordato D, Cathers I, Burne JA. An electromyographic study of parkinsonian swallowing and its response to levodopa. Mov Disord. 2012;27(14):1811-5.

23. Sundstedt S, Olofsson K, van Doorn J, Linder J, Nordh E, Blomstedt P. Swallowing function in Parkinson's patients following Zona Incerta deep brain stimulation. Acta Neurol Scand. 2012;126(5):350-6.

24. Kulneff L, Sundstedt S, Olofsson K, et al. Deep brain stimulation-effects on swallowing function in Parkinson's disease. Acta Neurol Scand. 2013;127(5):329-36.

25. Silbergleit AK, Lewitt P, Junn F, et al. Comparison of dysphagia before and after deep brain stimulation in Parkinson's disease. Mov Disord. 2012;27(14):1763-8.

26. Silbergleit AK, Schultz L, Jacobson BH, Beardsley T, Johnson AF. The Dysphagia handicap index: development and validation. Dysphagia. 2012;27(1):46-52.

27. • Carnaby-Mann G, Crary MA, Schmalfuss I, Amdur R. "Pharyngocise": randomized controlled trial of preventative exercises to maintain muscle structure and swallowing function during headand-neck chemoradiotherapy. Int J Radiat Oncol Biol Phys. 2012;83(1):210-219. A RCT compared "Pharyngocise" and usual dysphagia care with sham therapy and usual care in patients undergoing chemoradiation treatment for head and neck cancer. In secondary outcome measures of swallowing maintenance, there was a small benefit of "pharyngocise" over sham therapy.

28. • Manor Y, Mootanah R, Freud D, Giladi N, Cohen JT. Videoassisted swallowing therapy for patients with Parkinson's disease. Parkinsonism Relat Disord. 2013;19(2):207-211. An RCT compared conventional dysphagia therapy with and without videofeedback. This study uniquely provided effect sizes on conventional therapy alone. Video-feedback had a large effect size while conventional therapy alone yielded small effects on physiological measures of residue. Improvements in patients' perceptions of swallowing difficulty were moderate in the video-feedback group with no effects in the conventional group.

29. Manor Y, Giladi N, Cohen A, Fliss DM, Cohen JT. Validation of a swallowing disturbance questionnaire for detecting dysphagia in patients with Parkinson's disease. Mov Disord. 2007;22(13):1917-21.

30. McHorney CA, Martin-Harris B, Robbins J, Rosenbek J. Clinical validity of the SWAL-QOL and SWAL-CARE outcome tools with respect to bolus flow measures. Dysphagia. 2006;21(3):141-8.

31. Yang EJ, Baek SR, Shin J, et al. Effects of transcranial direct current stimulation (tDCS) on post-stroke dysphagia. Restor Neurol Neurosci. 2012;30(4):303-11.

32. •• Shigematsu T, Fujishima I, Ohno K. Transcranial direct current stimulation improves swallowing function in stroke patients. Neurorehabil Neural Repair. 2013;27(4):363-369. An RCT compared effects of tDCS paired with conventional dysphagia therapy with sham $t D C S$ paired with conventional dysphagia therapy at a mean of 3 months post-onset. Evaluation post-tDCS immediately following therapy had a large effect size while sham therapy had a small effect. One month post-treatment follow-up indicated that spontaneous recovery added further gains that were large in both groups.

33. Nam HS, Beom J, Oh BM, Han TR. Kinematic effects of hyolaryngeal electrical stimulation therapy on hyoid excursion and laryngeal elevation. Dysphagia. 2013. doi:10.1007/s00455-0139465-x.

34. Park JW, Kim Y, Oh JC, Lee HJ. Effortful swallowing training combined with electrical stimulation in post-stroke dysphagia: a randomized controlled study. Dysphagia. 2012;27(4):521-7. 
35. Baijens LWJ, Speyer R, Passos VL, et al. Surface electrical stimulation in dysphagic Parkinson patients: a randomized clinical trial. Laryngoscope. 2013. doi:10.1002/lary.24119.

36. Kushner DS, Peters K, Eroglu ST, Perless-Carroll M, Johnson-Greene D. Neuromuscular electrical stimulation efficacy in acute stroke feeding tube-dependent dysphagia during inpatient rehabilitation. Am J Phys Med Rehabil. 2013;92(6):486-95.

37. Kang JH, Park RY, Lee SJ, Kim JY, Yoon SR, Jung KI. The effect of bedside exercise program on stroke patients with dysphagia. Ann Rehabil Med. 2012;36(4):512-20.

38. Lan Y, Ohkubo M, Berretin-Felix G, Sia I, Carnaby-Mann GD, Crary MA. Normalization of temporal aspects of swallowing physiology after the McNeill dysphagia therapy program. Ann Otol Rhinol Laryngol. 2012;121(8):525-32.

39. Crary MA, Carnaby GD, Lagorio LA, Carvajal PJ. Functional and physiological outcomes from an exercise-based dysphagia therapy: a pilot investigation of the McNeill dysphagia therapy program. Arch Phys Med Rehabil. 2012;93(7):1173-8.
40. Moon TY, Kwon KH, Kim JW, et al. A study of functional dysphagia scale improvements after neuromuscular electrical stimulation. J Phys Ther Sci. 2013;25(1):61-4.

41. Sun SF, Hsu CW, Lin HS, et al. Combined neuromuscular electrical stimulation (NMES) with fiberoptic endoscopic evaluation of swallowing (FEES) and traditional swallowing rehabilitation in the treatment of stroke-related dysphagia. Dysphagia. 2013. doi:10.1007/s00455-013-9466-9.

42. Lee SY, Yang HE, Yang HS, Lee SH, Jeung HW, Park YO. Neuromuscular electrical stimulation therapy for dysphagia caused by Wilson's disease. Ann Rehabil Med. 2012;36(3):409-13.

43. Won YS. Influence of manual facilitation technique on swallowing disorder and aspiration pneumonia caused by severe dysphagia with stroke. J Phys Ther Sci. 2012;24(9):909-13.

44. O'Quigley J, Pepe M, Fisher L. Continual reassessment method: a practical design for phase 1 clinical trials in cancer. Biometrics. 1990;46(1):33-48.

45. Garrett-Mayer E. The continual reassessment method for dosefinding studies: a tutorial. Clin Trials. 2006;3(1):57-71. 